\title{
Primary Melanoma of the Lung Treated with Surgery, Dabrafenib and Trametinib
}

\author{
Layla G. Landgrafa Mark A. Malias ${ }^{b}$ Stephen J. Patterson ${ }^{c}$ \\ aDepartment of Clinical Research, Cleveland Clinic Indian River Hospital, Vero Beach, FL, \\ USA; ${ }^{b}$ Heart and Vascular Institute, Cleveland Clinic Indian River Hospital, Vero Beach, FL, \\ USA; 'Department of Oncology, Scully Welsh Cancer Center, Cleveland Clinic Indian River \\ Hospital, Vero Beach, FL, USA
}

Keywords

Primary lung melanoma Pulmonary melanoma $\cdot$ BRAF mutation V600K

\begin{abstract}
This is a case report on a 69-year-old woman who was diagnosed with malignant melanoma presenting in the lung. A complete staging workup was performed by her oncologist, with no skin lesion identified as a primary. CT-guided needle biopsy of the lung nodule was completed, with pathology revealing atypical cells consistent with malignant melanoma. Following full body examination by the cutaneous oncologist, a robotic wedge resection and lymph node dissection accompanied by bronchoscopy were performed. Molecular testing (Caris Life Sciences) of the surgical tumor specimen revealed pathological BRAF DNA mutation in exon 15/p. $V 600 K$. The patient was initially treated with nivolumab, then treated with a combination of dabrafenib and trametinib at recurrence.

\section{Introduction}

Malignant melanoma typically presents as a cutaneous primary tumor on the epidermis or mucous membranes. The primary presentation of a melanoma in the lung is exceedingly rare and poorly described in the literature. In addition, a definitive diagnosis is made difficult due to the lack of specific markers to differentiate lung primary tumor from metastasis. The results of thorough patient history-taking and lesion distribution assessment serve as diagnostic indicators in the absence of markers. 

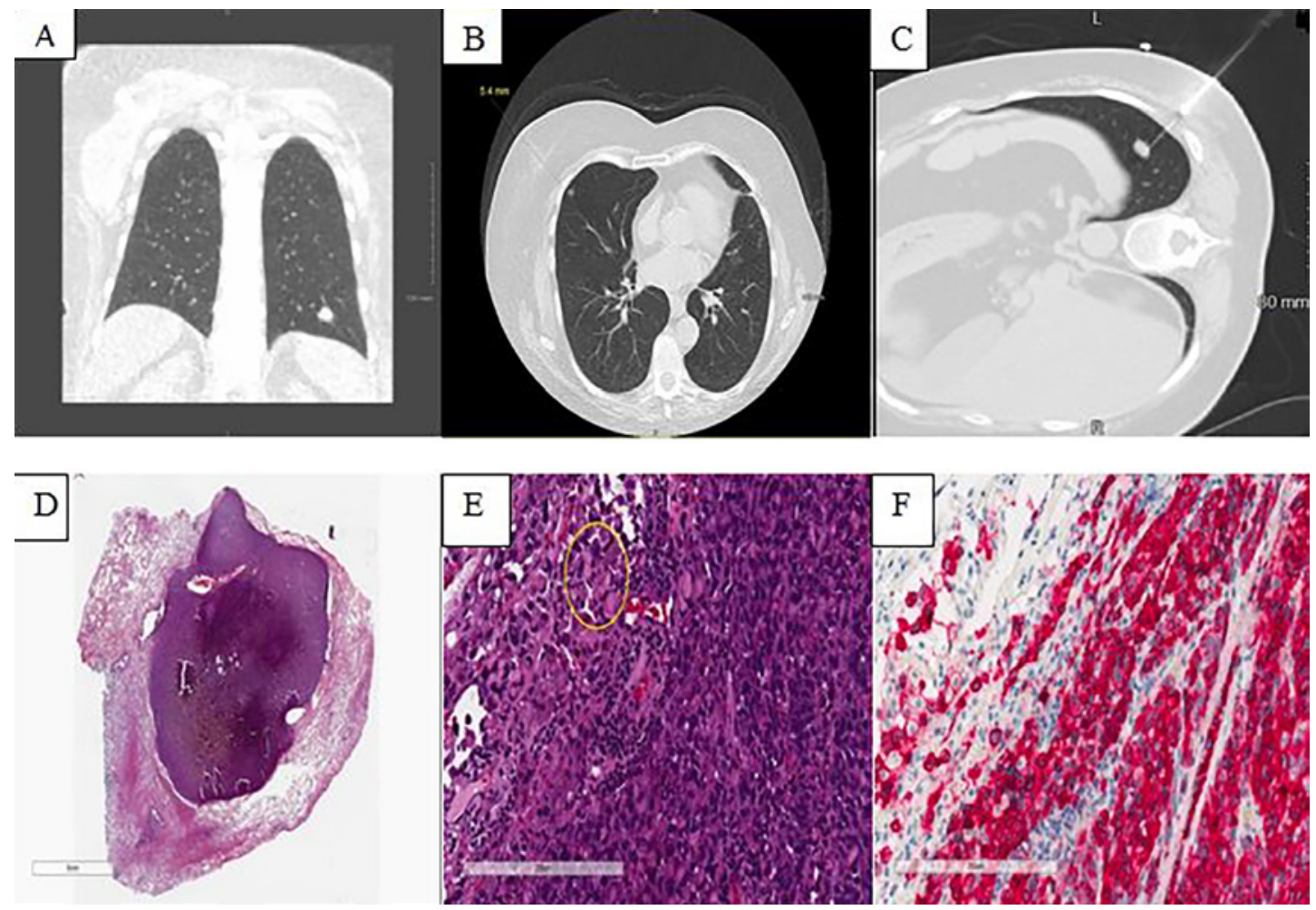

Fig. 1. A CT scan (coronal view) demonstrating a left lower lobe nodule (LLL; approx. $12 \mathrm{~mm}$ ). B CT scan (axial view) showing a right upper lobe nodule (approx. $5 \mathrm{~mm}$ ). C CT-guided biopsy of the LLL nodule. D H\&E stain indicating a fairly circumscribed, hypercellular, dark-stained nodule in the background of an unremarkable lung parenchyma (magnification, $\times 20$ ). E H\&E stain demonstrating plasmacytoid tumor cells with eccentrically located nuclei with abandoned eosinophilic cytoplasm (magnification, $\times 100$ ). F Melan-A staining (strongly positive) highlighting melanocytes (magnification, $\times 100$ ).

\section{Case Report}

A 69-year-old Caucasian female presented to her pulmonologist following an incidental finding of a 7-mm left lower lobe (LLL) lung nodule on magnetic resonance imaging (MRI) for a pancreatic duct stricture. The patient history included a left mastectomy and radiation therapy for breast cancer in 2000. There was no family history of melanoma or pancreatic cancer; however, both parents had died of lung cancer. The patient has been a lifetime nonsmoker and nondrinker, regularly exercised, and had no acute respiratory or constitutional complaints.

She returned to her pulmonologist after follow-up computed tomography (CT) scanning of the chest had demonstrated that the nodule had increased in size from 7 to $12 \mathrm{~mm}$, with a new 5-mm right upper lobe lung nodule 10 months later (Fig. 1A, B). Based on the rapid nodule growth and patient history, a CT-guided needle biopsy of the increasing LLL nodule was completed (Fig. 1C). Pathology revealed atypical cells with plasmacytoid morphology, with both H\&E staining (Fig. 1D, E) and melan-A staining (Fig. 1F) results consistent with malignant melanoma.

A complete staging workup was performed by the oncologist via MRI of the head and fullbody positron emission tomography (PET) scanning head to toe, but no skin lesion was identified as a primary. MRI of the head showed no enhancing mass lesion. PET revealed a very 
mild metabolism of the known malignant nodule in the posterior LLL with a standardized uptake value of 1.71. No other areas of concerning hypermetabolism were found on the headto-toe PET.

The patient was referred to a dermatologist for full-body examination of the skin and mucous membranes to rule out a primary melanoma lesion, with benign findings. Also, she was referred to an ophthalmologist to rule out retinal melanoma, with a negative result. The patient then was referred to a thoracic surgeon for resection. In view of additional right lung lesions, the surgeon opted for wedge resection of the LLL. The patient underwent robotic LLL wedge resection and lymph node dissection accompanied by bronchoscopy.

Surgical pathology revealed multiple foci of metastatic melanoma, the largest being $1.6 \times$ $1.5 \times 1.3 \mathrm{~cm}$, as well as 4 lymph nodes negative for melanoma. Molecular testing (Caris Life Sciences) of the surgical tumor specimen revealed pathological BRAF DNA mutation in exon 15/pV600K, PDGFRA mutation of unknown significance, and an intermediate tumor mutation burden at 16 mutations/Mb. No mutations were found in KIT, MEK1, MEK2, or NF1. Immunohistochemical staining found $M L H 1, M S H 2$, and $M S H 6$ positive at 2+, and $P D-L 1$ was negative.

The patient was scheduled for treatment with nivolumab at a flat dose of $480 \mathrm{mg}$ monthly for 12 months. However, the 6-month repeat PET/CT imaging demonstrated a significant increase in size of the right lobe nodule. In addition, new small pulmonary nodules were identified, with the largest in the left base measuring up to $6 \mathrm{~mm}$. The patient was not deemed to be a candidate for radiation. Treatment was changed from nivolumab to the two-drug combination of dabrafenib $150 \mathrm{mg}$ orally twice daily and trametinib $2 \mathrm{mg}$ once daily.

\section{Discussion}

Malignant melanoma arises from atypical melanocytes in the epidermis and often presents as a cutaneous tumor on the skin or mucous membranes [1]. Malignant melanoma presenting as a primary tumor in the lung accounts for only $1 \%$ of all melanoma cases [2]. Fewer than 45 cases of primary pulmonary malignant melanoma are reported in the literature, and they account for only $0.01 \%$ of all lung cancers $[3,4]$. Diagnosing primary lung melanoma or metastatic melanoma in the lung relies on the patient history and lesion distribution, as there are no specific markers to definitively differentiate a primary tumor from metastasis [5].

This case illustrates the relative paucity of data related to a confirmatory diagnosis of malignant melanoma presenting as a lung primary. Though it is indeed a rare presentation, it is not an impossibility, and must be considered when all available investigative methods fail to prove otherwise. A thorough history workup revealed no previous melanoma history or family history of melanoma, while both parents of the patient had died of lung cancer. The physical examinations by the dermatologist, oncologist, and ophthalmologist found no lesion on the skin, mucous membranes, or eyes, CT/PET imaging failed to identify any other lesion outside of the lungs, and surgical pathology revealed negative lymph node involvement.

Immunohistochemical staining of a melanoma tumor is not sufficient to prove its origin, and no specific molecular mutation is reported in the literature as being definitively diagnostic of pulmonary malignant melanoma. BRAF V600 mutation is not an unusual finding, occurring in approximately $50 \%$ of patients with melanoma [6]. However, most BRAF V600 mutations are $V 600 E$, with only $5 \%$ being V600K [7]. Altered PDGFRA is reported in $5.63 \%$ of melanoma cases [8]. No study has reported whether malignant pulmonary melanomas demonstrate BRAF V600 or PDGFRA mutations, or whether their presence or absence can indicate the origin of the disease. Further molecular studies are needed to characterize pulmonary melanoma in order to differentiate lung primary tumor from metastasis.

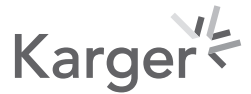




\section{Case Reports in Oncology}

\begin{tabular}{l|l}
\hline Case Rep Oncol 2020;13:789-792 \\
\hline DOI: 10.1159/000508093 & $\begin{array}{l}\text { @ 2020 The Author(s). Published by S. Karger AG, Basel } \\
\text { www.karger.com/cro }\end{array}$ \\
\hline
\end{tabular}

Landgraf et al.: Lung Melanoma Treated with Surgery and Immunotherapy

\section{Acknowledgements}

Special thanks are due to Mary Carter, PhD, for her review of the manuscript, to Shari Cole for providing the slide images, and to Dr. Julie Bell and Dr. Yunguang Liu for their expertise in slide review.

\section{Statement of Ethics}

Written informed consent was obtained from the patient for the publication of this case report and the accompanying images.

\section{Conflict of Interest Statement}

Stephen Patterson: Bristol-Myers Squibb consultant.

\section{Funding Sources}

The authors received no financial support for the authorship and/or publication of this case report.

\section{Author Contributions}

L.G.L. identified the case, obtained consent from the patient, and wrote the manuscript; M.A.M. wrote and edited the manuscript; S.J.P. diagnosed and treated the patient, and edited the manuscript.

\section{References}

1 Ward WH, Lambreton F, Goel N, Yu JQ, Farma JM. Clinical presentation and staging of melanoma. In: Ward WH, Farma JM, editors. Cutaneous melanoma: etiology and therapy [Internet]. Brisbane (AU): Codon Publications; 2017 Dec 21. Chapter 6. doi: 10.15586/codon.cutaneousmelanoma.

2 Ost D, Joseph C, Sogoloff H, Menezes G. Primary pulmonary melanoma: case report and literature review. Mayo Clin Proc. 1999;74(1):62-6.

3 Rodriguez FF, Uddin A, Nasr J. Primary pulmonary malignant melanoma found while evaluating new onset cough: a case presentation and literature review. Case Rep Pulmonol. 2019;2019:3867831.

4 Kyriakopoulos C, Zarkavelis G, Andrianopoulou A, Papoudou-Bai A, Stefanou D, Boussios S, et al. Primary pulmonary malignant melanoma: report of an important entity and literature review. Case Rep Oncol Med. 2017;2017:8654326.

5 Jagirdar J. Application of immunohistochemistry to the diagnosis of primary and metastatic carcinoma to the lung. Arch Pathol Lab Med. 2008;132(3):384-96.

6 Berger MF, Garraway LA. Applications of genomics in melanoma oncogene discovery. Hematol Oncol Clin North Am. 2009;23(3):397-414, vii.

7 Bradish JR, Cheng L. Molecular pathology of malignant melanoma: changing the clinical practice paradigm toward a personalized approach. Hum Pathol. 2014;45(7):1315-26.

8 AACR Project GENIE Consortium. AACR Project GENIE: powering precision medicine through an international consortium. Cancer Discov. 2017;7(8):818-31. 